\title{
Who are the important predators of sea turtle nests at Wreck Rock beach?
}

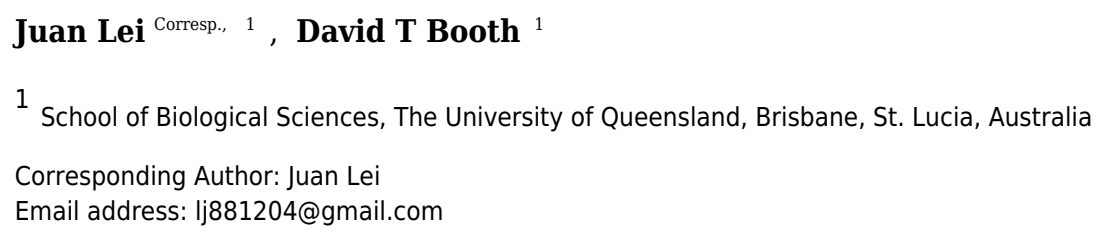

Excessive sea turtle nest predation is a problem for conservation management of sea turtle populations. This study assessed predation on nests of the endangered loggerhead sea turtle (Caretta caretta) at Wreck Rock beach adjacent to Deepwater National Park in Southeast Queensland, Australia after a control program for feral foxes was instigated. The presence of predators on the nesting dune was evaluated by tracking plots $(2 \times 1 \mathrm{~m}$ ) every $100 \mathrm{~m}$ along the dune front. There were 21 (2014-2015) and 41 (2015-2016) plots established along the dune, and these were monitored for predator tracks daily over three consecutive months in both nesting seasons. Predator activities at nests were also recorded by the presence of tracks on top of nests until hatchlings emerged. In addition, camera traps were set to record the predator activity around selected nests. The tracks of the fox (Vulpes vulpes) and goanna (Varanus spp) were found on tracking plots. Tracking plots, nest tracks and camera traps indicated goanna abundance varied strongly between years. Goannas were widely distributed along the beach and had a Passive Activity Index (PAI) (0.31 in 2014-2015 and 0.16 in 2015-2016) eight times higher than that of foxes (PAI 0.04 in 2014-2015 and 0.02 in 2015-2016). Five hundred and twenty goanna nest visitation events were recorded by tracks but no fox tracks were found at turtle nests. Camera trap data indicated that yellow-spotted goannas (Varanus panoptes) appeared at loggerhead turtle nests more frequently than lace monitors ( $V$. varius) did, and further that lace monitors only predated nests previously opened by yellow-spotted goannas. No foxes were recorded at nests with camera traps. This study suggests that large male yellowspotted goannas are the major predator of sea turtle nests at the Wreck Rock beach nesting aggregation and that goanna activity varies between years. 
1 Who are the important predators of sea turtle nests at Wreck Rock beach?

2 Juan Lei ${ }^{1}$ and David T. Booth ${ }^{1}$

$3{ }^{1}$ School of Biological Sciences, The University of Queensland, Brisbane, St. Lucia, QLD 4072,

4 Australia.

5 Corresponding Author:

6 Juan Lei ${ }^{1}$

$7 \quad$ Email: lj881204@gmail.com

8

9

10

11

12

13

14

15

16

17

18

19

20

21 
Abstract

Excessive sea turtle nest predation is a problem for conservation management of sea turtle

populations. This study assessed predation on nests of the endangered loggerhead sea turtle

(Caretta caretta) at Wreck Rock beach adjacent to Deepwater National Park in Southeast

Queensland, Australia after a control program for feral foxes was instigated. The presence of

predators on the nesting dune was evaluated by tracking plots $(2 \times 1 \mathrm{~m})$ every $100 \mathrm{~m}$ along the

dune front. There were 21 (2014-2015) and 41 (2015-2016) plots established along the dune,

and these were monitored for predator tracks daily over three consecutive months in both

nesting seasons. Predator activities at nests were also recorded by the presence of tracks on

top of nests until hatchlings emerged. In addition, camera traps were set to record the predator

activity around selected nests. The tracks of the fox (Vulpes vulpes) and goanna (Varanus spp)

were found on tracking plots. Tracking plots, nest tracks and camera traps indicated goanna

abundance varied strongly between years. Goannas were widely distributed along the beach

and had a Passive Activity Index (PAI) (0.31 in 2014-2015 and 0.16 in 2015-2016) eight times

higher than that of foxes (PAI 0.04 in 2014-2015 and 0.02 in 2015-2016). Five hundred and

twenty goanna nest visitation events were recorded by tracks but no fox tracks were found at

turtle nests. Camera trap data indicated that yellow-spotted goannas (Varanus panoptes) 
43 No foxes were recorded at nests with camera traps. This study suggests that large male yellow-

44 spotted goannas are the major predator of sea turtle nests at the Wreck Rock beach nesting aggregation and that goanna activity varies between years.

Introduction

Sea turtles are oviparous and construct their nests on dunes adjacent to the beach where embryos take about two month to incubate. Sea turtle hatchling nest emergence success is determined by nest temperature, salinity, humidity, water inundation and predation (Fowler 1979; Miller 1985; Reid et al. 2009). During incubation, a wide range of predators may attack sea turtle nests and have a significant effect on hatchling recruitment and thus long-term population persistence (Stancyk 1995). At many beaches nest predation is the main cause of hatch failure of sea turtles with some regions reporting more than $50 \%$ of nests being 
64

introduced fox (Vulpes vulpes), pig (Sus scrofa) and wild dog (Canis familaris) (Limpus 1978;

Limpus \& Fleay 1983). In particular, fox predation of sea turtle nests along the east Australian coast has been problematic and therefore a major focus of sea turtle conservation programs (Limpus 1978; Limpus \& Fleay 1983; Limpus 2008).

The loggerhead turtle (Caretta caretta) is an endangered species on the IUCN Red List (IUCN 2016). Major breeding aggregations of loggerhead sea turtle include Africa-Mozambique, Oman, the Mediterranean sea, Sri Lanka, Japan, U.S.A. and Australia (Limpus \& Limpus 2003). Genetic studies indicate there is little or no interbreeding between these major breeding aggregations (Bowen et al. 1993; Limpus 2008). In Australia, two genetically distinct breeding stocks have been identified: an eastern Australian population and western Australian population (Limpus \& Limpus 2003). If one breeding stock becomes extinct, it would be difficult to repopulate this area from other genetic stocks. In order to preserve the genetic diversity of loggerheads, it is necessary to protect each of the different populations.

A significant number of loggerhead turtles nest at Wreck Rock beach adjacent to Deepwater National Park, Queensland, Australia ( 400 nests per season, Limpus 2008). Predators of sea turtle nests at Wreck Rock beach include foxes, dingoes and goannas (Limpus 2008). The fox predation of loggerhead turtle nests continued to increase from a modest level when monitoring commenced in 1968-1969 to 90-95\% in the mid-1970s (Limpus 2008). From 1987 onwards, 1080 poison baits have been used to control fox predation (Limpus 2008), but a 
recent nest survey (McLachlan et al. 2015) indicated that while fox predation of nests was minimal, a large number of nests were predated by goannas. The lace monitor (Varanus varius) and yellow-spotted goanna (Varanus panoptes) are likely to be the main goannas attacking loggerhead nests because of their distribution along the coastline and ability to dig holes while foraging (Cogger 1993). However, the relative activity levels and impact of these species on loggerhead turtle nests at Wreck Rock beach remain unknown.

For some animal species, it is difficult to estimate population density by standard census methods such a mark and recapture (Engeman \& Allen 2000) because of large home ranges, rough terrain habitats, relatively sparse populations and/or difficulty in capturing animals or making direct observations (Pelton and Marcum 1977). To overcome these problems, Engeman \& Allen (2000) developed and refined a passive activity index (PAI) based on the occurrence of tracks on small, pre-defined plots of substrate for monitoring wild carnivore species. This method is simple and quickly applied in the field and can also provide accurate information reflecting population changes over time or space, and simultaneously capture a suite of wildlife species (Engeman \& Allen 2000). This method has been used previously to monitor predator activities, including the common water monitor (Varanus salvator) activity on an olive ridley turtle (Lepidochelys olivacea) nesting beach in Indonesia over two nesting seasons (Maulany 2012). 
106

107

108

109

110

111

112

113

114

number of sea turtle nests at Wreck Rock beach (Limpus 2008; McLachlan et al. 2015), no

quantitative study of sea turtle nest predation has been conducted at this important nesting

beach, and it is not known what species of goanna is responsible for predation. Therefore, the aim of this study was to fill this knowledge gap by quantifying goanna and fox activity on nesting dunes during the sea turtle nesting season at Wreck Rock beach. Three methods were used to achieve this aim. Firstly, tracking plots were used to monitor general activity levels of goannas and foxes along the dunes where sea turtles construct their nests. Secondly, turtle nests were inspected every day until turtle hatchlings emerged in order to record the activities of predators at the nest. Thirdly, camera traps were used to capture predator activity at sea turtle nests so that we could identify which species of goanna was the main predator of these nests.

\section{Methods}

Study site and nest marking

This study was conducted along the beach for $3 \mathrm{~km}$ immediately to the north and south of Wreck Rock adjacent to Deepwater National Park, Southeast Queensland (24 $18^{\prime} 58$ S, $151^{\circ} 57^{\prime}$ 55" E) (Fig. 1). This section of the beach is marked by numbered stakes every $100 \mathrm{~m}$ for ease of marking and relocating nests. The beach was monitored nightly by personnel from Turtle Care Volunteers Queensland Inc. to record the presence of emerging female turtles and successful nesting activities. When a nest was located, its position was marked by a red ribbon attached to a small stake and recorded using a handheld GPS (Garmin eTrex 30, Kansas, USA). All work was 
127

128

129

130

131

132

133

134

135

approved by a University of Queensland Animal Ethics Committee (permit \#SBS/352/EHP/URG) and conducted under Queensland Government National parks scientific permit \# WITK15315614.

Tracking plots

Tracking plots were used to estimate relative activity of predators during the peak sea turtle nesting time across two consecutive years (5-Dec-2014 to 4-Mar-2015 and 30-Nov-2015 to 28Feb-2016). In 2015-2016, these plots were also monitored for four days in April, a time when most sea turtle clutches had finished hatching. Twenty-one tracking plots (2 m x $1 \mathrm{~m})$ in 20142015 and 41 in 2015-2016, spaced 100 m apart, were set up on the primary dune (where most sea turtle nests were constructed). The plots extended along the dunes for $1 \mathrm{~km}$ (2014-2015) and $2 \mathrm{~km}$ (2015-2016) north and south of Wreck Rock camping area. The monitored area of a plot was marked by sticks placed at each corner of the plot and the plot's location was recorded with a handheld GPS. Each plot was inspected daily during the afternoon (weather permitting), and the number of goanna and fox tracks recorded. After reading, plots were resurfaced using a rake to obliterate tracks, insuring the same tracks were not recorded on subsequent days. The activity of predators was quantified using the passive activity index (PAI) of Engeman et al. (1998):

$P A I=\frac{1}{d} \sum_{j=1}^{d} \frac{1}{P j} \sum_{i=1}^{P j} X i j$ where the Xij value represents the number of tracking plot tracks by an observed species at the ith plot on the jth day; $d$ is the number of days of inspection, and $P j$ is the number of plots 
148

contributing data on the $j$ th day. PAI was calculated for each day throughout the study for statistical comparisons, and at 10-day intervals for graphical presentation of data. Because PAI data failed a Kolmogorov-Sminov normally test, K-S d = 0.223, $p<0.01, n=320$; a nonparametric Kruskal-Wallis ANOVA by Ranks followed by a multiple comparison test was used to test for inter-species and inter-year differences in nest visitation rates.

\section{Nest monitoring}

Once a nest was located it was visited daily throughout the incubation period in order to identify predation events and the tracks of animals visiting nests. Each nest was inspected during the morning (weather permitting) and the number of goanna and fox tracks was recorded. Nest area approximately $1 \mathrm{~m}^{2}$ was resurfaced by using a rake after observation. Nest visitation rate was quantified as a percentage by dividing the number of days fresh tracks were found at a nest by the total number of nest inspection days (nest inspection days = total number of times a nest was inspected during the season until hatchlings emerged from the nest or until it was totally predated). Because goanna nest visitation data failed a KolmogorovSminov normally test, $\mathrm{K}-\mathrm{S} d=0.1442, \mathrm{p}<0.05, \mathrm{n}=121$; a non-parametric Kolmogorov-Sminov two sample test was used to test for inter-season differences in nest visitation rates.

\section{Camera traps}

Camera traps (Reconyx Hyperfire HC600, Holmen, Wisconsin, USA) were set up to capture images of predators visiting a sample of 12 loggerhead turtle nests (randomly selected) 
169

170

171

between 6 December 2014 and 27 January 2015 and 30 nests (randomly selected) between 1 December 2015 and 27 February 2016. Camera traps were at each nest for 25 days in 2014-

2015 and 30 days in 2015-2016. All camera traps were triggered by motion sensors and could be triggered 24 hours per day. Camera traps were positioned $50 \mathrm{~cm}$ behind the selected turtle nests, at least $30 \mathrm{~cm}$ above ground. Each camera trap had a $1 \mathrm{~m}^{2}$ field of view over the nest insuring that any nest visitation by predators was recorded. This enabled information on the frequency, time of day and species to be collected. To compare the relative activity of goannas visiting nests each year and between years, we calculated the per-nest per-day visitation rate for camera trap monitored nests. For each nest the number of independent images (defined as taken at least 20 minutes apart; multiple images taken within 20 minutes of each other were classified as a single visitation event) of goannas recorded at that nest was divided by the number of days the camera was set at that nest. Because camera trap per-nest per-day data failed a Kolmogorov-Sminov normally test, K-S $d=0.292, p<0.01, n=84$; a non-parametric Kruskal-Wallis ANOVA by Ranks followed by a multiple comparison test was used to test for inter-species and inter-year differences in nest visitation rates. Circular statistics were used to analyze the time of day that nests were visited by yellow-spotted goannas and lace monitors.

Non-parametric statistical analysis was performed using Statistica Ver 13.1 (Dell Inc.) software, and circular statistical analysis was performed using Oriana Ver 4 (Kovach Computing Services).

Results 
190 Tracking plots

191 Monitored tracking plots revealed tracks of two potential egg predators, goannas (lace

192 monitors and yellow-spotted goannas combined as it was not possible to distinguish between

193 the two species on the basis of their tracks alone) and foxes. Only a few dog tracks were

194 identified in tracking plots during the course of the study. However, these dog tracks were most

195 likely made by pet dogs accompanying tourists visiting the beach, and so have been excluded

196 from analysis.

197

During the first nesting season (5-Dec-2014 until 4-Mar-2015), 21 plots were monitored for 71

days with 466 goanna and 62 fox occurrences recorded (Table 1). During the second nesting

200

season (5-Dec-2015 until 28-Feb-2016), 41 plots were monitored for 89 days with 535 goanna

201

and 70 fox occurrences recorded (Table 1). There were differences in occurrence rates detected

between goannas and foxes and years (Table 1), Kruskal-Wallis test: $H(3, n=320)=180.065, p$

$<0.001$. Multiple comparison tests indicated that goanna activity was approximately 7 times

greater than fox activity in both seasons, and goanna activity in 2014-2015 was approximately

twice that in 2015-2016, but fox activity was not different between the two seasons. During the

2014-2015 nesting season, goanna activity on the dune front remained relatively constant

throughout the season (Fig. 2). Fox activity was generally much lower than goanna activity from

December through January, but there was a conspicuous increase in fox activity in February (Fig.

2). In the $2015-2016$ nesting season, goanna activity was relatively low in December, increased 
211 April at a time when most sea turtle nests had hatched. Fox activity remained low and relatively

212 constant throughout the entire season (Fig. 2).

213

214 Nest monitoring

215 During the 2014-2015 nesting season, 52 loggerhead turtle nests were monitored, and $57.7 \%$ of

216 these nests were predated by goannas as indicated by burrows constructed into the nest egg

217 chamber. During the 2015-2016 nesting season, 46 nests were monitored, and $17.4 \%$ of these

nests were predated by goannas. No fox or other predators were observed to raid turtle nest in

either season. During 2014-2015, 520 goanna nest visits (lace monitors and yellow-spotted

goannas combined as it was not possible to distinguish between the two species on the basis of

their tracks alone) as were recorded while in 2015-2016, 343 nest visits were recorded (Table 2).

Daily per-nest visits were significantly greater in 2014-2015 than in 2015-2016 (Kolmogorov-

Smirnov two sample test, $p<0.001$ ) being approximately two times greater (Table 2$)$. Nests

that were predated could be dug open for the first time at any time during the incubation

period; there was no trend for the first nest attack to be associated with nest construction or

nest hatching (Fig. 3).

Camera traps

Images from camera traps showed that goannas were the only predators to visit monitored

nests; no images of foxes or wild dogs were recorded. All of the monitored nests had at least 
232

233

234

235

236

237

238

recorded in the 2014-2015 nesting season, 47 (85.5\%) of these visitation events were made by yellow-spotted goannas and only $8(14.5 \%)$ were made by lace monitors (Table 3$)$. The overall per-nest per-day visitation rate was 0.157 for yellow-spotted goannas and 0.027 for lace monitors (Table 3). Despite all camera traps being deployed by 20 December 2014, only two goannas appeared at nests in December 2014, but activity at nests increased sharply from the beginning of January 2015 (Fig. 4a). Eggs were seen to be consumed on 17 occasions (14 yellow-spotted goannas, 3 lace monitors). Yellow-spotted goannas were seen to open a nest for the first time on 17 occasions, but lace monitors were only ever seen to visit nests that had already been opened. In the 2015-2016 nesting season, 107 goanna nest visiting events were captured, $87(81.3 \%)$ of these visitation events were made by yellow-spotted goannas and only $20(18.7 \%)$ were made by lace monitors (Table 3$)$. The overall per-nest per-day visitation rate was 0.097 for yellow-spotted goannas and 0.022 for lace monitors (Table 3). Eggs were seen to be predated by yellow-spotted goanna on 6 occasions. No lace monitors were seen consuming eggs in this season. There were difference in visitations rates between monitored groups, Kruskal-Wallis test: $\mathrm{H}(3, \mathrm{n}=84)=26.826, \mathrm{p}<0.001$; with multiple comparison tests indicating that yellow-spotted goanna visitation rate was greater than lace monitors in both seasons, but there was no difference in inter-season visitation rate in either species.

Goannas visited nests at any time of the day between 8:00 and 18:00 (Fig. 4a,b). Combining data from both seasons, and plotting the data separately for yellow-spotted goannas and lace monitors revealed that yellow-spotted goannas had a bi-modal nest visitation pattern, with a 
253

254

255

256

257

258

259

260

261

262

263

264

265

266

267

268

269

270

271

272

273

peak in activity in the morning between 7:00 and 11:00, 8:57 \pm 1:10 (mean \pm circular standard deviation) and again in the afternoon between 13:00 and 16:00, 15:10 $\pm 0: 50$, while the most frequent time for visits from lace monitors was in the afternoon the late afternoon between 15:00 and 17:00, 15:46 \pm 0.50 (Fig. 5). A Watson-Williams F-test $\left(F_{1,154}=11.792, p<0.001\right)$ confirmed that when considering all nest visitation data, the mean time of lace monitor visits $(13: 38 \pm 2: 53, n=29)$ was later than yellow-spotted goanna visits $(11: 27 \pm 3: 00, n=129)$.

An entire nest opening sequence was recorded on 23 Jan 2015. A large yellow-spotted goanna first began digging at 14:12 (Fig 6a). It reached the egg chamber and consumed the first egg at 14:28 after 16 minutes of continuous digging activity (Fig 6b). Turtle eggs were swallowed intact, one at a time, by the goanna rather than being opened and having their contents licked out (Fig $6 c)$. This goanna stopped feeding and left the nest at 16:56 after almost 2.5 hours of feeding and having consumed eight eggs.

\section{Discussion}

Nest predation decreases the recruitment of hatchlings and has become an important challenge for the conservation of egg-laying reptiles (Leighton et al. 2010). Hence, understanding the activity of predators adjacent to endangered reptilian species breeding aggregations is important for designing conservation strategies. The daily checking for predator tracks on nests and the deployment of tracking plots and camera traps allowed us to continuously monitor activities of nest predators adjacent to a loggerhead turtle nesting beach. There were two 
274

275

276

277

278

279

280

281

282

significant results from the study that provide new insights into goanna predation of sea turtle nests. First, camera trap data indicated that yellow-spotted goannas are the most frequent visitors and predators of sea turtle nests at Wreck Rock beach and were the only species observed to open nests, suggesting they are the main cause of nest predation. Second, the nest predation rate and activity of goannas on the nesting dune varied by a factor of two between the two seasons that we studied.

\section{Predator activities at nests}

Camera traps allowed us to explore the loggerhead turtle nest predator species, predation time and behavior of predators while at nests. Yellow-spotted goannas were the most frequent visitors and predators of sea turtle nests in this study. Large adult yellow-spotted goannas have the ability to dig up sea turtle nests and swallow turtle eggs intact, suggesting future management strategies should be targeted at these individuals. Indeed, no lace monitors were observed to open sea turtle nests directly, they were only observed predating nests that had already been opened by yellow-spotted goannas. Hence, lace monitors appear to be opportunistic nest predators on this beach. Lace monitors are frequently arboreal and are equipped with long, recurved claws that facilitate climbing (Cogger 1993). Such claws are not particularly useful for digging and may explain why they did not open nests. Using GPS tracking methodology, Lei \& Booth (2015) reported yellow-spotted goannas use the beach dunes more than lace monitors and are therefore more likely to predate sea turtle nests than lace monitors. Moreover, camera traps did not record foxes at nests, and no fox tracks were observed over 
295

296

297

298

299

300

301

302

303

304

305

306

307

308

309

310

311

312

313

314

315

nests during this study indicating that the fox baiting program deployed by park managers is

currently effective at inhibiting fox predation of sea turtle nests at Wreck Rock beach.

Although camera trap records indicated that sea turtle nests were visited by yellow-spotted goannas at any time of day between 7:00 and 17:30, visits were most frequent in the morning and afternoon with a distinct lull during the middle of the day. This reflects the general activity pattern of yellow-spotted goannas as recorded by GPS tracking data (Lei et al. 2017). It would appear that the midday heat suppresses the foraging activity of yellow-spotted goannas, and this may be particularly so in the beach dune area there are no trees to provide shade. In contrast, although the data is far less numerous, lace monitors had a single peak in sea turtle nest visiting activity, and this was late in the afternoon, typically after the peak afternoon yellow-spotted goanna nest visiting time. Hence, lace monitors may arrange their nest visiting times to avoid interacting with yellow-spotted goannas. Further investigation of this possibility is needed.

Doody et al. $(2014,2015)$ reported that yellow-spotted goannas can dig warren complexes that required removal of sand from up to $3 \mathrm{~m}$ deep and that both males and females contribute to warren excavation. Hence, the job of digging into a sea turtle nest which is comparatively shallow (40 - $80 \mathrm{~cm}$ ), should be relatively easy as evidenced by it requiring only 16 minutes of digging to gain access to eggs in one of our monitored nests. Our camera trap images indicated that yellow-spotted goannas normally dug into the nest at an angle from one side of the nest to 
reach the nest chamber rather than digging a hole vertically downwards from directly above the nest. Hence, when covering a nest with mesh as a management strategy used to deter nest predation, the mesh must be relatively large in area (at least $1 \times 1 \mathrm{~m}$ ) to prevent yellow-spotted goanna burrowing into the nest (Lei \& Booth 2017). Turtle nest predation rate is likely dependent on cues left by the female turtle (e.g. visual, tactile, and olfactory), and many

predators have the ability to detect these cues (Vander Wall 1998, 2000; Geluso 2005; Leighton

et al. 2009). Goannas use their forked tongue to transfer olfactory cues to the specialized chemosensory Jacobson's organ and so are adept at using olfactory cues to find prey (Blamires

\& Guinea 1998; King \& Green 1999; Vincent \& Wilson 1999). We found that once a turtle nest was opened, this nest was continually predated over subsequent days by multiple yellowspotted goannas.

We suspected that goannas might attack sea turtle nests more frequently immediately after their construction, or after hatching at the end of incubation. These expectations were based on the idea that sand disturbance and the smell of the female and or newly laid eggs around the sand might give clear clues to foraging goannas immediately after nest construction, and that the smell of egg fluids released during the hatching process might also attract goannas at the end of incubation. This was not what we observed; a nest was equally likely to be attacked for the first time at any time during incubation. We do not know why this is the case, particularly as goannas crawled over the top of some nests several times during incubation without attacking them, and then at a later date these nests were attacked. One possibility 
337

338

339

340

341

342

343

344

345

346

347

348

might be that ghost crabs (Ocypode ceratophthalmus and $O$. cordimanus) which are numerous on the nesting beach and frequently burrow into sea turtle nests, cause the release of 'incubating egg odor' which then attracts goannas.

Predator activity

Based on the PAI analysis of tracking plot data, the activity of goannas was higher than that of foxes, suggesting that goannas are the main predator of sea turtle nests at Wreck Rock beach, a conclusion also supported by nest track and camera trap data. We found that all of our monitored nests were visited by goannas and that between $17 \%$ (2015-2016) and 58 \% (20142015) of nests were opened by yellow-spotted goannas. Goanna predation of nests had previously been reported as greater than $50 \%$ at this beach (McLachlan et al. 2015). It is unclear whether goanna predation of sea turtle nests was this high at Wreck Rock beach during preEuropean settlement times or whether more recent perturbations have led to increased nest predation in recent times. During the 1970s-1990s goanna predation of sea turtle nests at this location was not detected, but fox predation of nests was high, $90 \%$ of nests being predated in the 1970's and up until 1987 (Limpus 2008). From 1987 onwards, a fox baiting program reduced fox predation on sea turtle nests to negligible levels (Limpus 2008). Goanna predation of sea turtle nests was first reported in the 2003-2004 nesting season when two nests were predated (Limpus 2008), and since then goanna predation of sea turtle nests has increased so that over $50 \%$ of sea turtle nests were being attacked by goannas in the 2013-2014 season (McLachlan et al. 2015). Hence, the reduction in fox numbers may have also resulted in an increased 
358

359

360

361

362

363

364

recruitment of yellow-spotted goannas (because red foxes probably also predated yellowspotted goanna nests) to historically high levels. However, before European settlement and the introduction of foxes, hunting of goannas by native people may have kept the density of goannas on the frontal dunes at a low level.

Goanna activity in 2014-2015 was twice as high as in 2015-2016, as was the nest predation rate. This suggests that nest predation is positively correlated with goanna activity. Maulany (2012) reported that olive ridley turtle nests suffered $100 \%$ predation by monitor lizards at a beach adjacent to Alas Purwo National Park, Banyuwangi (East Java), Indonesia, which had high monitor lizard activity (PAI = 1.27 in 2009, 1.41 in 2010). This finding also suggests that goanna activity on dunes is a good predictor of intensity of goanna predation on sea turtle nests.

Fox activity increased at the end of the 2014-2015 nesting season. Typically the park mangers fox bait twice during the sea turtle nesting season, once in early December and again in early February. In 2014-2015 the February baiting was missed, so any foxes that might have moved into the beach area after the December baiting were not removed. However, in the 2015-2016 season, the early February fox baiting probably maintained fox activity at low levels.

The goanna predation rate of sea turtle nests in 2014-2015 was twice that in 2015-2016, and it correlated with an increase in goanna activity on the dune. The nest visitation rate by recording tracks on nests in 2014-2015 was nearly twice that in 2015-2016. These results suggested 
379

380

381

382

383

384

385

386

387

388

389

390

391

392

393

394

395

396

397

398

399

goanna activity on the dune in 2014-2015 was higher than in 2015-2016. However, it remains

unclear why goanna activity and sea turtle nest predation rate varied so greatly between the

two nesting seasons. Because of the strong inter-annual differences in predator indices over

two years, additional years of research are needed to determine the long-term average

predation rate and its implications for turtle hatching success.

Implications for management

Lei \& Booth (2017) compared different methods of directly protecting sea turtle nests against goanna predation and found that deploying the plastic mesh on the top of turtle nests was the most effective and economic way. Combined with our observations of digging behaviour of yellow-spotted goanna captured on camera traps, we suggested that plastic mesh needs to be at least $1 \times 1 \mathrm{~m}$ to prevent yellow-spotted goannas digging into the nest chamber. In addition, camera trap data indicated turtle nest predation activities happen any time between 7:00 and $17: 00$, suggesting turtle nest management should be deployed in the early morning following the night that nests are constructed. More management strategies such as temporary removal of large male yellow-spotted goannas or egg relocation should be investigated in the future to counteract the loss of sea turtle nests to yellow-spotted goanna predation.

\section{Acknowledgements}

This work would not have been possible without the help of Nev and Bev McLachlan's Turtle Care Volunteers organization, the Burnett Mary Regional Group and WWF Australia. 
References

402

Blamires SJ, Guinea ML. 1998. Implications of nest site selection on egg predation at the sea

403

turtle rookery at Fog Bay. Proceedings of Marine Turtle Conservation and Management in

Northern Australia Workshop, Darwin, 20-24.

405

406

Blamires SJ, Guinea ML, Prince RIT. 2003. Influence of nest site selection on predation of

flatback sea turtle (Natator depressus) eggs by varanid lizards in Northern Australia. Chelonian

Conservation and Biology 4: 557-563.

409

410

Bowen BW, Richardson JI, Meylan AB, Margaritoulis D, Hopkins-Murphy SR, Avise J. 1993.

411

Population structure of loggerhead turtles (Caretta caretta) in the west Atlantic Ocean and

Mediterranean Sea. Conservation Biology. 7: 834-844.

Brown L, Macdonald DW. 1995. Predation on green turtle Chelonia mydas nests by wild canids

at Akyatan Beach, Turkey. Biological Conservation 71: 55-60.

416

417

Cogger H. 1993. Reptiles and amphibians of Australia. Sydney: A. H. \& A. W. Reed.

Doody JS, James H, Ellis R, Gibson N, Raven M, Mahony S, Hamilton DG, Rhind D, Clulow S,

McHenry CR. 2014. Cryptic and complex nesting in the yellow-spotted monitor, Varanus 
panoptes. Journal of Herpetology 48: 363-370.

422

423 Doody SJ, James H, Colyvas K, McHenery CR, Clullow S. 2015. Deep nesting in a lizard, deja vu 424 devil's corkscrews: first helical reptile burrow and deepest vertebrate nest. Biological Journal of the Linnean Society 116: 13-26.

Engeman RM, Allen L, Zerbe GO. 1998. Variance estimate for the Allen activity index. Wildlife

Research 25: 643-648.

429

Engeman RM, Allen L. 2000. Overview of a passive tracking index for monitoring wild canids and

associated species. Integrated Pest Management Reviews 5: 197-2003.

Fowler LE. 1979. Hatching success and nest predation in the green sea turtle, Chelonia mydas,

at Tortuguero, Costa Rica. Ecology 60: 946-955.

Frick MG. 2003. The surf crab (Arenaeus cribrarhs): A predator and prey item of sea turtles.

Marine Turtle Newsletter 99: 16-18.

438

Geluso K. 2005. Benefits of small-sized caches for scatter-hoarding rodents: influence of cache size, depth, and soil moisture. Journal of Mammal 86: 1186-1192. 
442 IUCN. 2016. 2016 IUCN Red List of threatened species. Available from: http://www.redlist.org/

443

444 King D, Green B. 1999. Monitor: the biology of varanid lizards. Sydney: NSW University Press.

445

446 Lei J, Booth DT. 2015. The use of GPS logging devices and camera traps to track goanna

447 movement on and adjacent to a south east Queensland sea turtle rookery. Reef, Range and Red

Dust Conference, Caloundra Queensland August 2015, 17.

449

450

Lei J, Booth DT. 2017. How best to protect the nests of the endangered loggerhead turtle

Caretta caretta from monitor lizard predation? Chelonian Conservation and Biology (in press).

Lei J, Booth DT, Dwyer RG. 2017. Spatial ecology of yellow-spotted goannas adjacent to a sea

turtle nesting beach. Australian Journal of Zoology (in press).

Leighton PA, Horrocks JA, Krueger BH, Beggs JA, Kramer DL. 2008. Predicting species

interactions from edge responses: mongoose predation on hawksbill sea turtle nests in

fragmented beach habitat. Proceedings of the Royal Society B 275: 2465-2472.

Leighton PA, Horrocks JA, Kramer DL. 2009. How depth alters detection and capture of buried

prey: exploitation of sea turtle eggs by mongooses. Behavioral Ecology 20: 1299-1306. 
463 Leighton PA, Horrocks JA, Kramer DL. 2010. Predicting nest survival in sea turtles: when and 464 where are eggs most vulnerable to predation? Animal Conservation 14: 186-195.

465

466 Limpus CJ. 1978. The reef: uncertain land of plenty. In: Lavery HJ. ed. Exploration North:

467 Australia's Wildlife from Desert to Reef, Richmond Hill Press, 187-222.

468

469

Limpus CJ, Fleay A. 1983. Management and turtles. Proceedings of the Great Barrier Reef

Conference Townsville, James Cook University Press, 535-540.

Limpus CJ, Limpus DJ. 2003. Loggerhead turtles in the Equatorial and Southern Pacific Ocean: a species in decline. In: Bolten AB, Witherington BE ed. Loggerhead Sea Turtles, Smithsonian Institution Press, 199-209.

Limpus CJ. 2008. A biology review of Australian marine turtles. 1. Loggerhead turtle, Caretta caretta (Linneaus). Queensland Government Environmental Protection Agency.

Maulany RI. 2012. The Nesting Biology, Ecology, and Management of the Olive Ridley Turtle

Thesis, The University of Queensland, Australia.

McLachlan N, McLachlan B, Hof C, Giudice S, Shuster G, Bunce A, Limpus C, Eguchi T. 2015. 
484 Predator reduction strategies for protecting loggerhead turtle nests at Wreck Rock beach in

485

486

487

488

489

490

491

492

493

494

495

496

497

498

499

500

501

502

503

504

Queensland. Reef to Range and Red Dust Conference, Caloundra Queensland August 2015, 15.

Miller JD. 1985. Embryology of marine turtle. In: Gans C, Billett F, Maderson PFA, ed. Biology of the Reptilia Wiley-Interscience, New York, 269-328.

Mora JM, Robinson DC. 1984. Predation of sea turtle eggs Lepidochelys by the snake Loxocemus

bicolor Cope. Revista de biologia tropical 32: 161-162.

Pelton MR, Marcum LC. 1977. The potential use of radioisotopes for determining densities of black bears and other carnivores. Proceedings of 1975 Predator Symposium pp: 221-236.

Reid K, Margaritoulis D, Speakman JR. 2009. Incubation temperature and energy expenditure during development in loggerhead sea turtle embryos. Journal of Experimental Marine Biology and Ecology 378: 62-68.

Stancyk SE, Talbert OR, and Dean JM. 1980. Nesting activity of the loggerhead turtle Caretta caretta in South Carolina, II. Protection of nests from raccoon predation by transplantation.

Biological Conservation 18: 289-298.

Stancyk SE. 1995. Non-human predators of sea turtles and their control. In: Bjorndal KA, ed. 
505 Biology and Conservation of Sea Turtles Washington, DC: Smithsonian Institution Press, 139-151.

506

507

508 Vander Wall SB. 1998. Foraging success of granivorous rodents: effects of variation in seed and

509 soil water on olfaction. Ecology 79: 233-241.

510

511 Vander Wall SB. 2000. The influence of environmental conditions on cache recovery and cache

512 pilferage by yellow pine chipmunks (Tamias amoenus) and deer mice (Peromyscus maniculatus).

513 Behavioral Ecology 11: 544-549.

514

515 Vincent M, Wilson S. 1999. Australian goannas. New Holland Publishers Pty Ltd, Sydney, NSW. 


\section{Table $\mathbf{1}$ (on next page)}

Table 1. Nest visitation events by tracking plots

Passive activity index (PAI) of goannas and foxes from dune tracking plots during the 2014-2015 and 20152016 sea turtle nesting seasons at Wreck Rock beach. 
1 Table 1. Passive activity index (PAI) of goannas and foxes from dune tracking plots during the 2 2014-2015 and 2015-2016 sea turtle nesting seasons at Wreck Rock beach.

\begin{tabular}{|c|c|c|}
\hline Nesting season & 2014-2015 & $2015-2016$ \\
\hline Plots monitored & 21 & 41 \\
\hline Monitored days & 71 & 89 \\
\hline \multicolumn{3}{|l|}{ Goannas } \\
\hline Total events recorded & 466 & 535 \\
\hline \multicolumn{3}{|l|}{ Daily PAI } \\
\hline mean \pm SD & $0.313 \pm 0.217$ & $0.150 \pm 0.104$ \\
\hline median, $25 \%$ - $75 \%$ quartile & $0.286,0.143-0.423$ & $0.125,0.075-0.200$ \\
\hline range & $0.000-1.048$ & $0.000-0.500$ \\
\hline \multicolumn{3}{|l|}{ Foxes } \\
\hline Total events recorded & 62 & 70 \\
\hline \multicolumn{3}{|l|}{ Daily PAI } \\
\hline mean $\pm S D$ & $0.042 \pm 0.079$ & $0.020 \pm 0.033$ \\
\hline median, $25 \%$ - $75 \%$ quartile & $0.000,0.000-0.048$ & $0.000,0.000-0.025$ \\
\hline range & $0.000-0.381$ & $0.000-0.175$ \\
\hline
\end{tabular}




\section{Table 2 (on next page)}

Visitation rate of goannas based on tracks found on top of nest

Visitation rate of goannas (based on tracks found on top of nests) at loggerhead turtle nests (visits per-nest per-day) for the 2014-2015 and 2015-2016 sea turtle nesting seasons at Wreck Rock beach. 
1 Table 2. Visitation rate of goannas (based on tracks found on top of nests) at loggerhead turtle

2 nests (visits per-nest per-day) for the 2014-2015 and 2015-2016 sea turtle nesting seasons at

3 Wreck Rock beach.

\begin{tabular}{lcc}
\hline \multicolumn{1}{c}{ Nesting season } & $2014-2015$ & $2015-2016$ \\
\hline Nest monitored & 52 & 46 \\
Monitored days & 41 & 80 \\
\hline Total events recorded & 520 & 343 \\
Daily visitation rate & & $0.110 \pm 0.107$ \\
mean \pm SD & $0.336 \pm 0.187$ & $0.087,0.022-0.175$ \\
median, $25 \%-75 \%$ quartile & $0.312,0.212-0.476$ & $0.000-0.455$ \\
range & $0.000-0.882$ & \\
\hline
\end{tabular}

4 


\section{Table 3(on next page)}

Nest visitation rate by camera traps

The nest visitation rate (per-nest per-day) recorded by camera traps at nests during 20142015 and 2015-2016 sea turtle nesting seasons at Wreck Rock beach. 
1 Table 3. The nest visitation rate (per-nest per-day) recorded by camera traps at nests during 2 2014-2015 and 2015-2016 sea turtle nesting seasons at Wreck Rock beach.

\begin{tabular}{|c|c|c|}
\hline Nesting season & 2014-2015 & $2015-2016$ \\
\hline Nests monitored & 12 & 30 \\
\hline Monitored days & 25 & 30 \\
\hline \multicolumn{3}{|l|}{ Yellow-spotted goannas } \\
\hline Total events recorded & 47 & 87 \\
\hline \multicolumn{3}{|l|}{ Visitation rate } \\
\hline mean \pm SD & $0.157 \pm 0.247$ & $0.097 \pm 0.116$ \\
\hline median, $25 \%$ - $75 \%$ quartile & $0.060,0.040-0.160$ & $0.050,0.033-0.133$ \\
\hline range & $0.040-0.920$ & $0.000-0.467$ \\
\hline \multicolumn{3}{|l|}{ lace monitors } \\
\hline Total events recorded & 8 & 20 \\
\hline \multicolumn{3}{|c|}{ Visitation events per nest per day } \\
\hline mean $\pm S D$ & $0.027 \pm 0.049$ & $0.022 \pm 0.029$ \\
\hline median, $25 \%$ - $75 \%$ quartile & $0.000,0.000-0.040$ & $0.000,0.000-0.033$ \\
\hline range & $0.000-0.160$ & $0.000-0.100$ \\
\hline
\end{tabular}


Figure 1 (on next page)

Figure 1. Image of study area

A: Location of study site, Wreck Rock beach adjacent to Deepwater National Park, Queensland, Australia. B: The locations of the loggerhead turtle nests monitored during the 2014-2015 nesting season. C: The locations of the loggerhead turtle nests monitored during the 2015-2016 nesting season. Shaded grey area indicates the section of beach monitored in this study. 

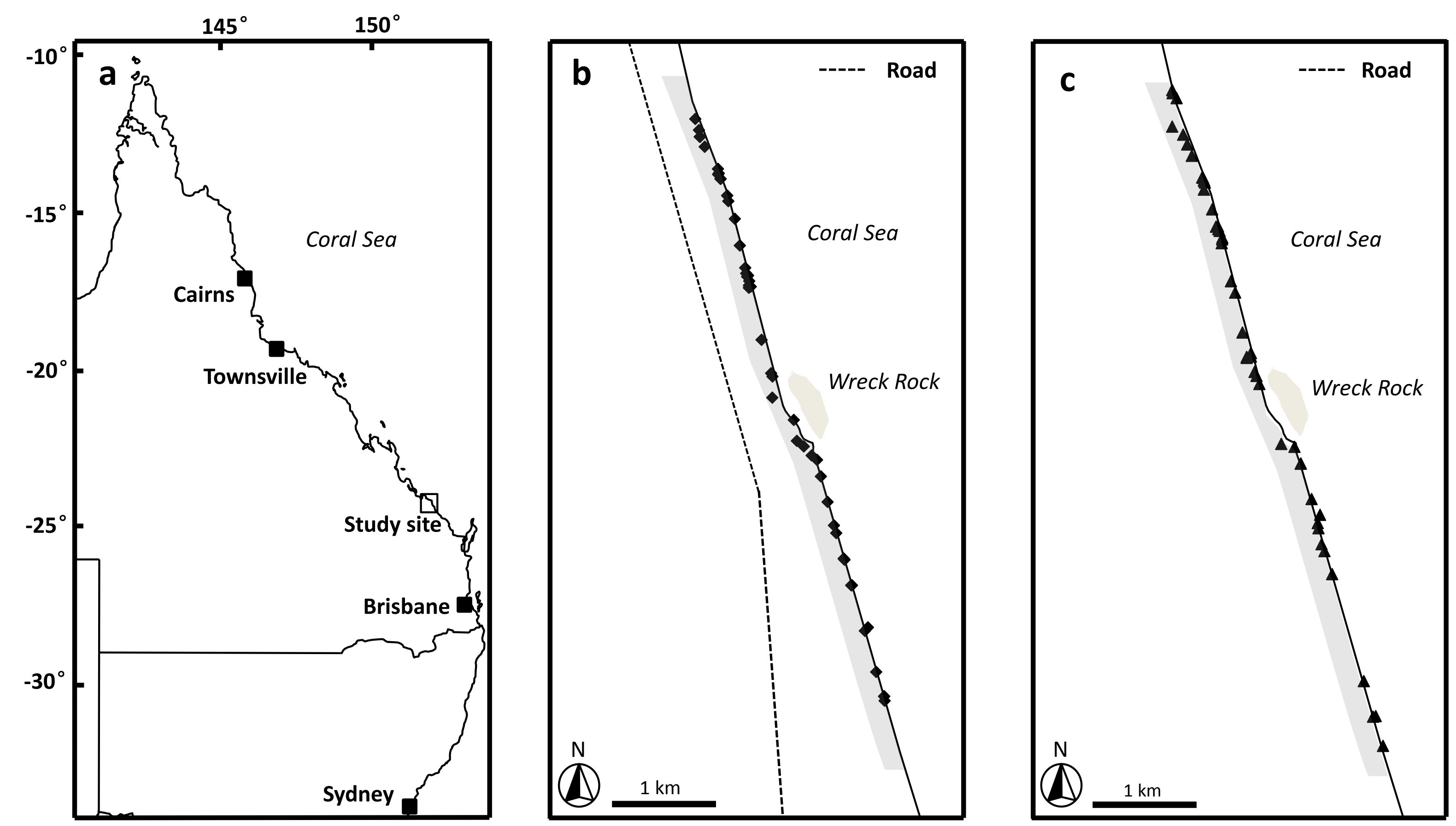


\section{Figure 2 (on next page)}

Figure 2. Figure of nest predator activity index (PAI)

Passive activity index (PAl, mean \pm SD of 10-day intervals) on the front dune at Wreck Rock Beach during the A) 2014-2015 and B) 2015-2016 nesting seasons for goannas (diamonds) and foxes (triangles). Data represent the center of 10-day means. 


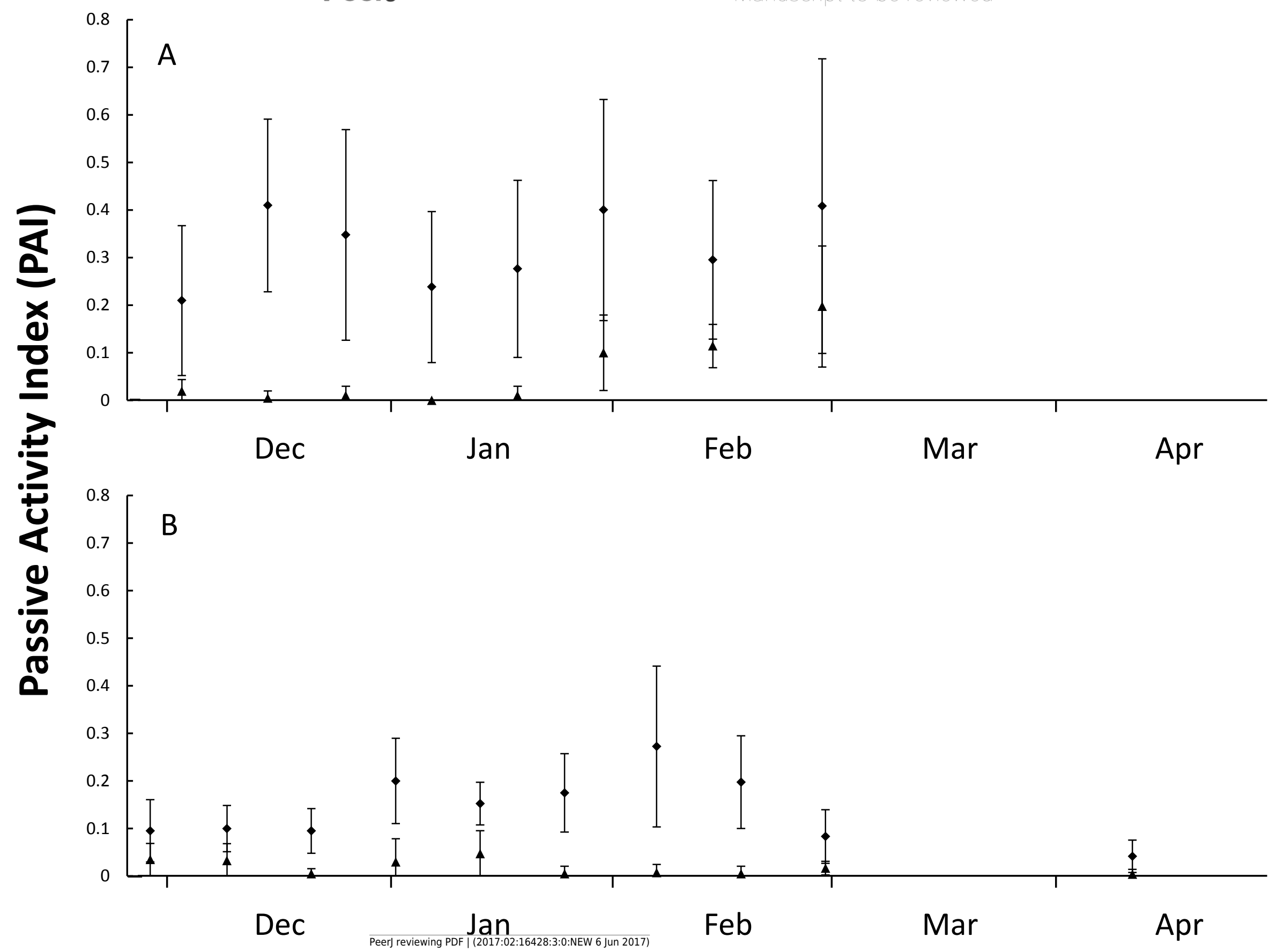




\section{Figure 3 (on next page)}

Figure 3. Plot of the frequency of nest predation events against the time since nest construction and first goanna predation event for loggerhead nests laid

Frequency of goanna first predation events in relation to the time since nest construction for loggerhead nests laid during the 2014-2015 (solid diamonds) and 2015-2016 (open triangles) nesting seasons at Wreck Rock beach. 


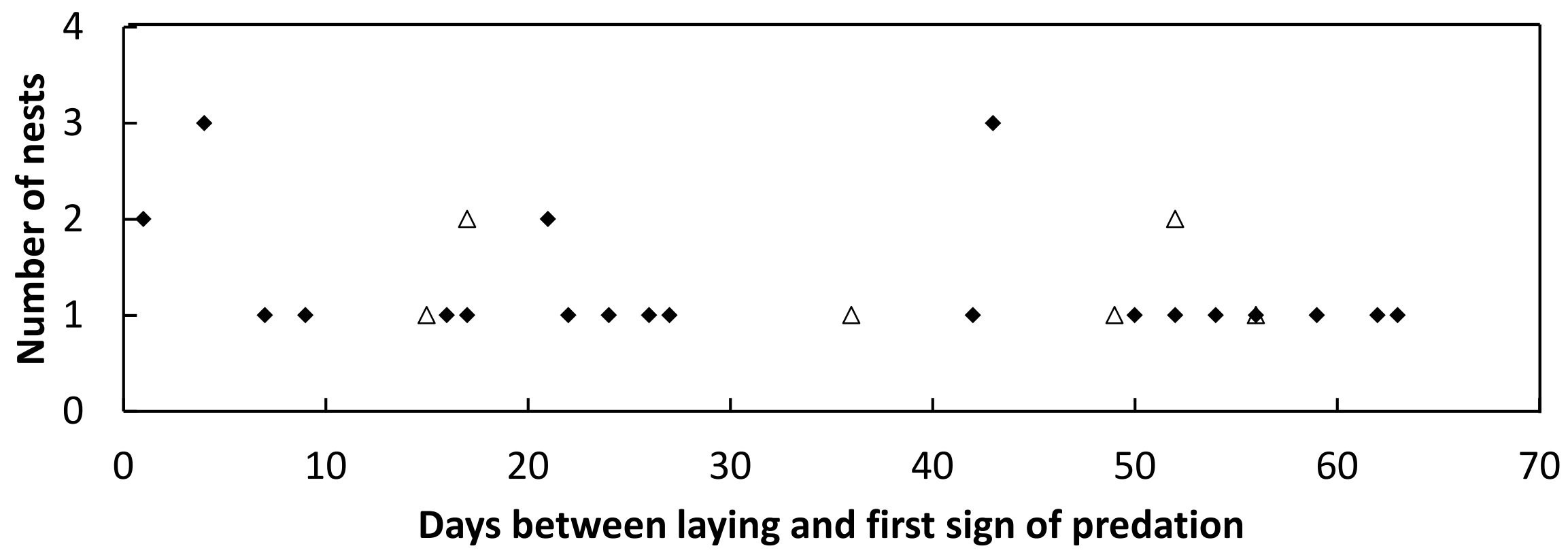


Figure 4 (on next page)

Figure 4. A figure of predators' activity on the turtle nesting beach Figure of time and date of goanna appearances at loggerhead turtle nests as determined from camera trap records

Time and date of goanna appearances at loggerhead turtle nests as determined from camera trap records. Triangle $=$ yellow-spotted goannas, Diamond $=$ lace monitors. A. Three hundred camera days (12 cameras set for 25 days each) during the 2014-2015 season. B. Nine hundred camera days (30 cameras set for 30 days each) during the 2015-2016 season. 


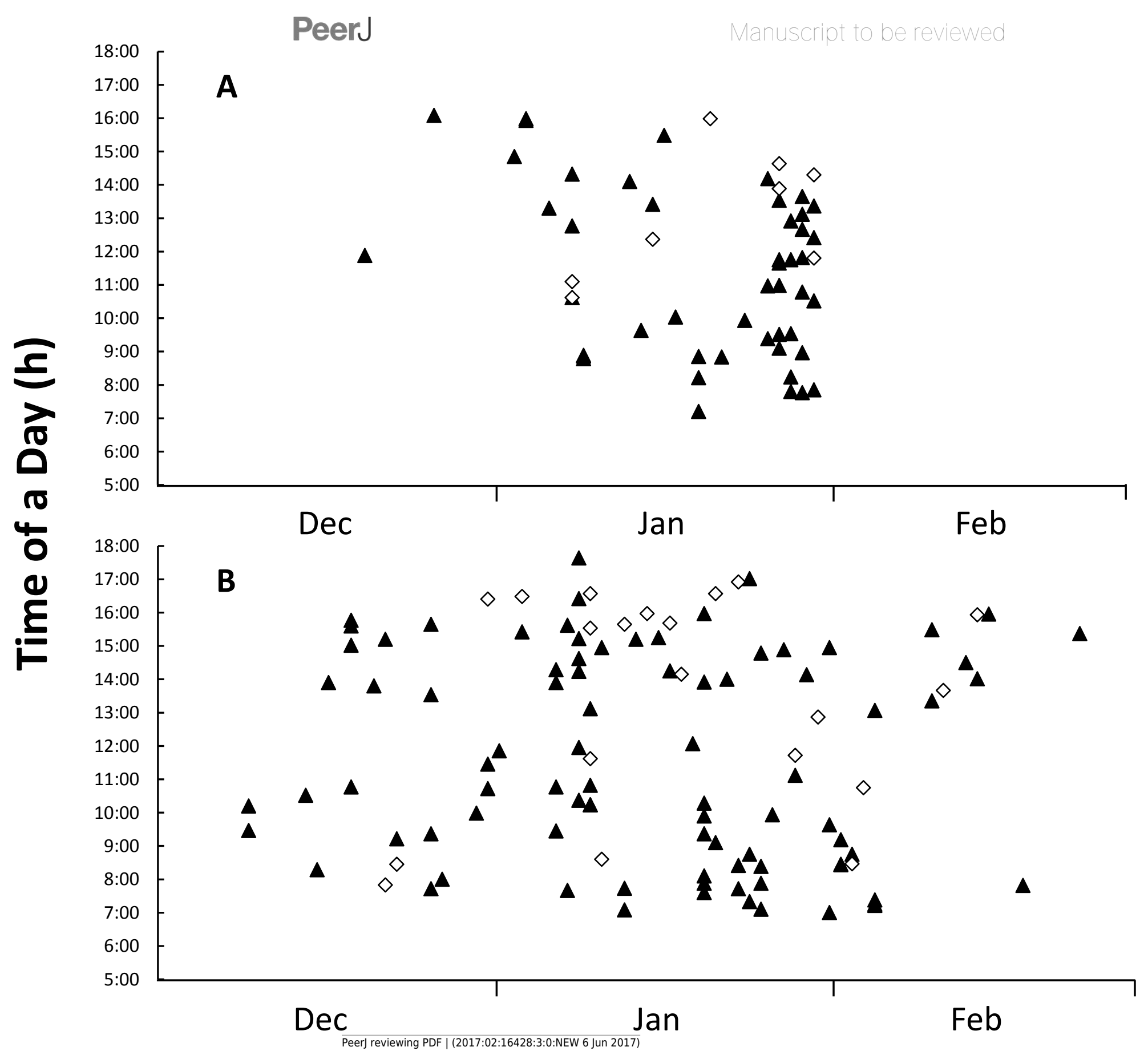




\section{Figure $\mathbf{5}$ (on next page)}

Figure 5. Plot of the number of images of goannas against time of day

The number of images of goannas taken by camera traps set at loggerhead turtle nests at Wreck Rock beach in relation to time of day that images were recorded. 


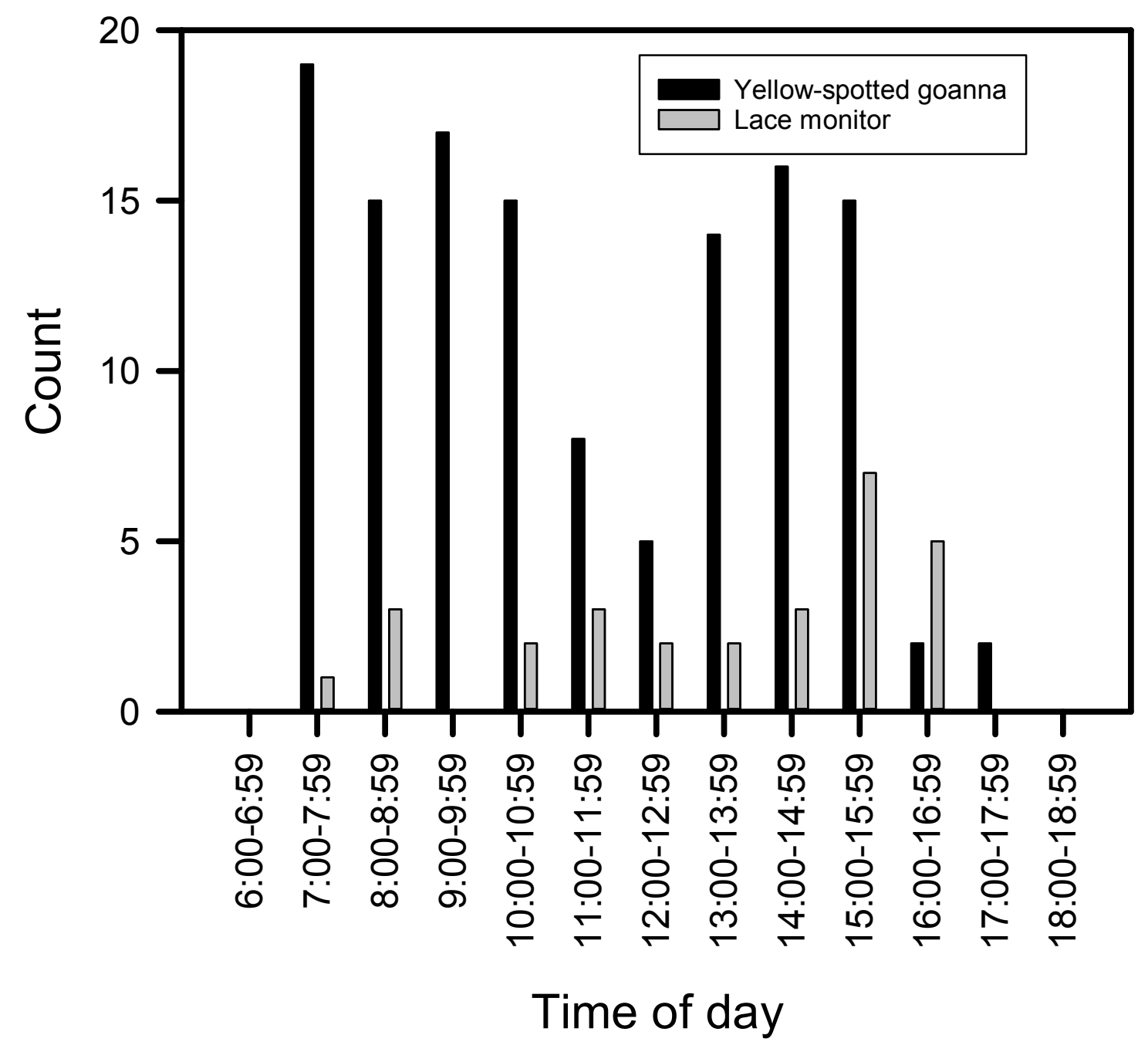




\section{Figure 6}

Figure 6. The photos of a yellow-spotted goanna opening and consuming eggs from a loggerhead turtle nest

A Yellow-spotted goanna opening and consuming eggs from a loggerhead turtle nest on 23-Jan-2015. Photos were captured by a camera trap. a. Start of digging, b \& c, removal and consumption of the first egg. For full sequence, see video in the supplementary information. 

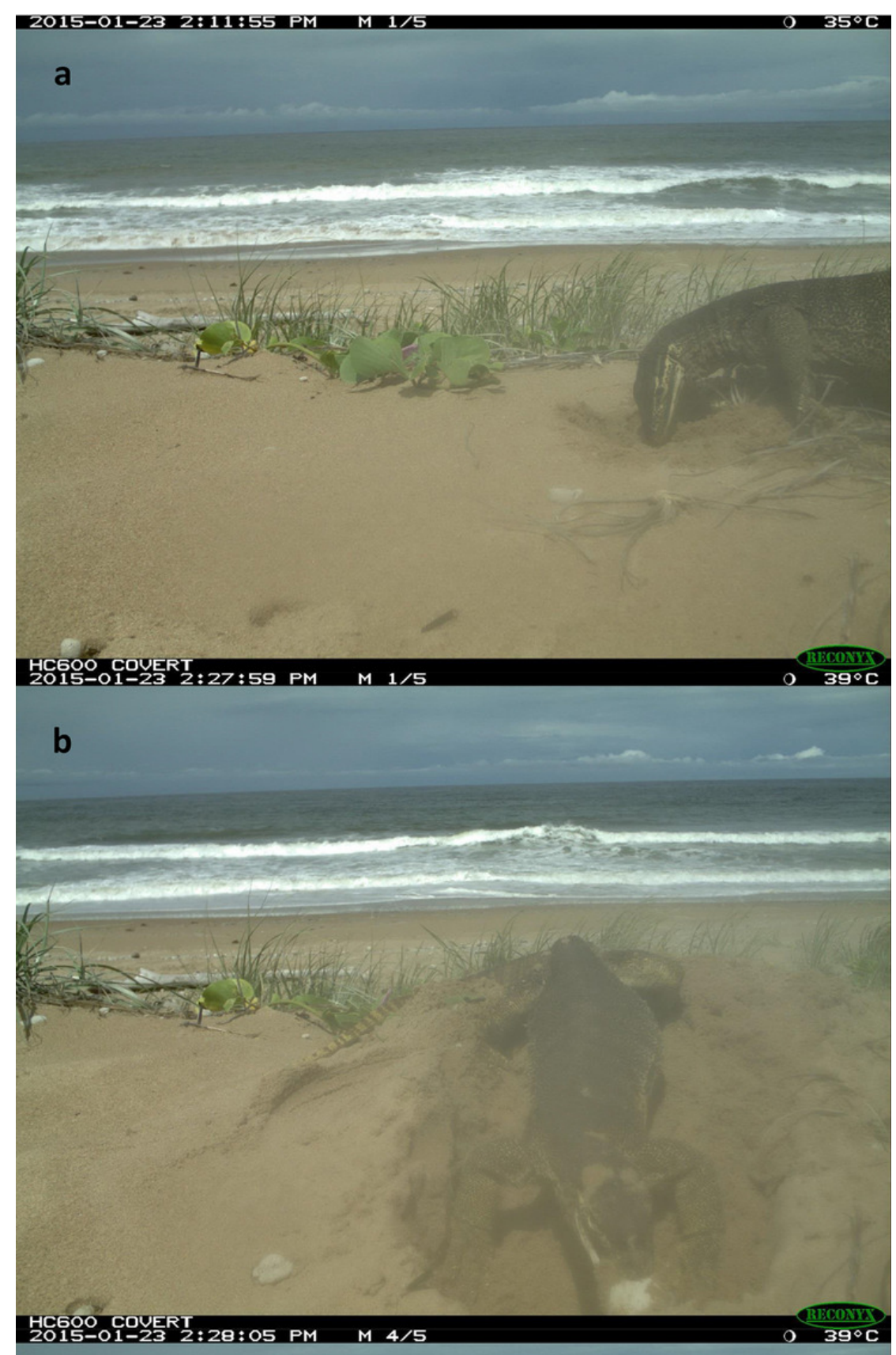

C

PeerJ reviewing PDF | (2017:02:16428:3:0:NEW 6 Jun 2017) 